\title{
CONTEXTUAL INTERFERENCE EFFECTS ON MOTOR SKILL ACQUISITION, RETENTION AND TRANSFER IN SPORT RIFLE SHOOTING
}

original paper

( ) University School of Physical Education in Wroclaw

DOI: https://doi.org/10.5114/hm.2018.74065

\section{NELSON ALEXANDRE MORETTO, ALEXANDRE JEHAN MARCORI, VICTOR HUGO ALVES OKAZAKI}

Londrina State University, Londrina, Brazil

\begin{abstract}
Purpose. Motor learning is the response to a new experience or practice of a skill that results in the production of a new motor skill. The contextual interference (CI) effect is a learning effect which describes the benefits of interference during practice as improved skill retention and skill transfer. Though it is an established phenomenon, the efficacy of CI has not yet been proven in complex motor tasks. Therefore, the purpose of this study was to analyze the CI effect on motor skill acquisition, retention, and transfer in sport rifle shooting.

Methods. 32 subjects were equally divided into two practice groups: high contextual interference (HCI) and low contextual interference (LCI). Four blocks of thirty shots were performed 10 meters from the target. Three positions (standing, sitting, and lying down) were used to manipulate the CI effect. The HCI group changed positions before each shot, while the LCI group shot 10 times in the same position before changing to the next one. All conditions were randomized between groups and subjects. One week after the 120 acquisition shots, retention and transfer (15 and 25 meters from the target) tests were carried out. Results. Accuracy between groups during the acquisition phase, retention test results, and transfer test results were similar between groups. Therefore, the CI effect was not observed in any of the phases of motor learning in sport rifle shooting.

Conclusions. It is possible these results are associated with the amount of practice, level of CI used, and complex characteristic of the task.
\end{abstract}

Key words: random practice, blocked practice, motor learning, motor skills

\section{Introduction}

Motor learning field studies evaluate methods and procedures that facilitate and enhance the skill acquisition process. The contextual interference (CI) effect is an interesting tool for this purpose. According to Shea and Morgan [1], practice with higher CI, or increased variability, allows superior learning and transfer compared to practice with lower CI. This variability is related to practice organization which can be manipulated to decrease the predictability of the factors involved in successfully executing the novel motor skill.

Many studies have been conducted to identify the optimal level of contextual interference that best aids skill acquisition. Wu and colleagues [2] found greater performance and learning with higher CI practice in a laboratory task which required only elbow joint movement. Similarly, both Meira, Fairbrother and Perez [3] and Paulwels, Swinnen and Beets [4] reported better retention levels of a motor skill when practice varied more despite poorer initial performance during the acquisition phase.

On the other hand, Carter and Grahn [5] did not observe greater outcomes with varied practice when analyzing the CI effect on learning to play a musical concert with a clarinet. Neville and Trempe's [6] research showed that random practice hinders new motor skill consolidation, claiming that high variability between tasks interferes in learning. Furthermore, a systematic review about motor learning of surgical and medical

Correspondence address: Alexandre Jehan Marcori, Universidade Estadual de Londrina, Rua Roberto Júlio Roehrig, 320, 86047-090 Londrina, Brazil, e-mail: alexandremarcori@gmail.com

Received: October 21, 2017

Accepted for publication: December 14, 2017

Citation: Moretto NA, Marcori AJ, Okazaki VHA. Contextual interference effects on motor skill acquisition, retention and transfer in sport rifle shooting. Hum Mov. 2018;19(2):99-104; https://doi.org/10.5114/hm.2018.74065. 
related tasks [7] revealed no significant difference in outcomes between higher and lower CI practice organization groups.

Brady [8], in a literature review of the contextual interference effect on sport skill, points out the diminished effect of CI manipulation on non-laboratory motor tasks. In his paper, Brady brought up evidence that the CI effect, as proposed initially by Shea and Morgan [1], is stronger when testing simple skills with less practical and applied characteristics. Therefore, a specific amount of CI may be appropriate for learning each motor skill. Analyzing different skill classes, then, is of great importance to better understand and apply the CI phenomenon. Little attention has been given to analyzing precision-based tasks such as the rifle shot. Despite its Olympic status and popularity as a leisure activity in some cultures, little research has addressed comprehension of the motor learning processes of this skill.

Rifle shooting can be considered a complex motor skill due to the challenge it presents to motor control. As proposed by Tuller, Turvey and Fitch [9], when trying to aim a gun the subject's body is not completely motionless. Therefore, the aim of the gun sways around the target constantly. If there is a target 10 meters away, for example, and 30-degrees is the perfect absolute angle of the shoulder joint to shoot, a 31-degree shot would lead to a $23 \mathrm{~cm}$ bullet displacement. The complexity of this task, then, revolves mostly around controlling many degrees of freedom of the arm segment, although other body parts are also involved in a way that a minimal change of position in any joint angle should be corrected by a compensatory movement of another joint(s) to achieve a successful shot. In other words, a shooter must constrain his muscles and joints to behave as a single coordinative structure [9].

The CI effect on motor learning in sport rifle shooting has not been documented in the literature. Therefore, the aim of this study is to analyze the CI effect on motor skill acquisition, retention, and transfer in sport rifle shooting. We hypothesized that the lower CI group (LCI) would demonstrate better initial performance, while the higher CI group (HCI) would demonstrate better retention and transfer levels. The findings in this study may contribute to understanding the appropriate CI dosage to apply to sport rifle shooting motor skill acquisition and enhance our knowledge about the CI effect on a complex motor task.

\section{Material and methods}

\section{Participants}

Thirty-two subjects (26 men, 6 women, age range 18 to 32 years old) with no previous experience in shooting volunteered to participate in this study. The subjects were divided into two groups, one group with high CI during practice and the other with low CI. The groups each contained 16 participants and equal number of men and women. Participants were allowed to use their preferred hands, and all of them self-declared right-hand preference for this and other manual tasks.

\section{Experimental procedures}

Contextual interference was manipulated by varying the shooting positions which were standing (ST), sitting field target style (FT), and lying down (LD), as shown in figure 1 . In all conditions, participants were not allowed to support the rifle on any place or object except their own body.

The acquisition phase consisted of a total of 120 shots, 10 meters from the target, blocked in 4 sets of 30 shots each. Within each block, participants shot 5 times at the same target followed by a two-minute rest. The HCI group varied the shooting position with every shot, while the LCI group shot 10 times before changing positions. The position order was randomized between subjects of each group.

After a week without any practice, retention and

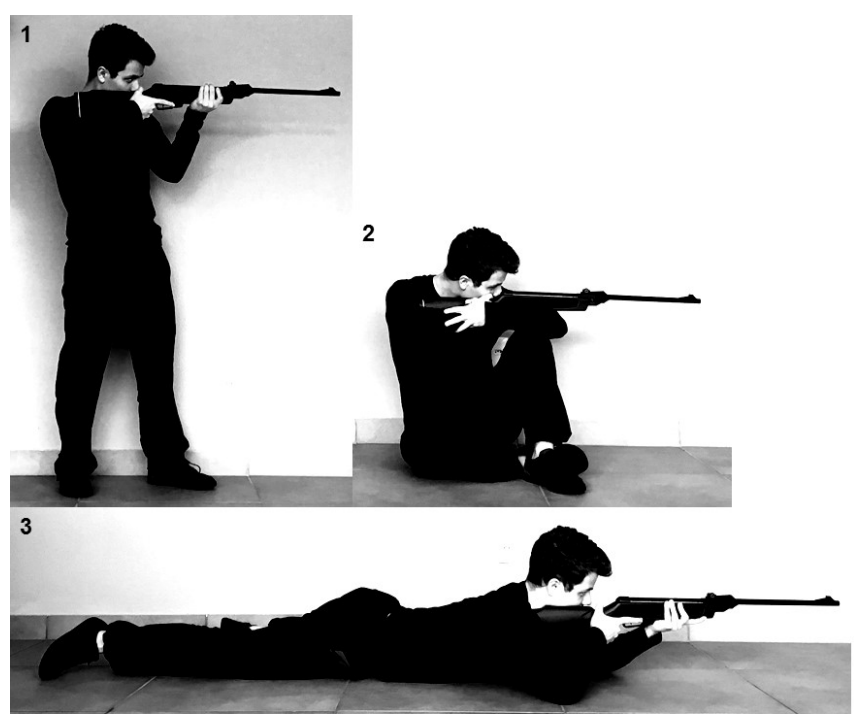

Figure 1. Shooting position representation: standing (1), sitting field target style (2), and lying down (3) 


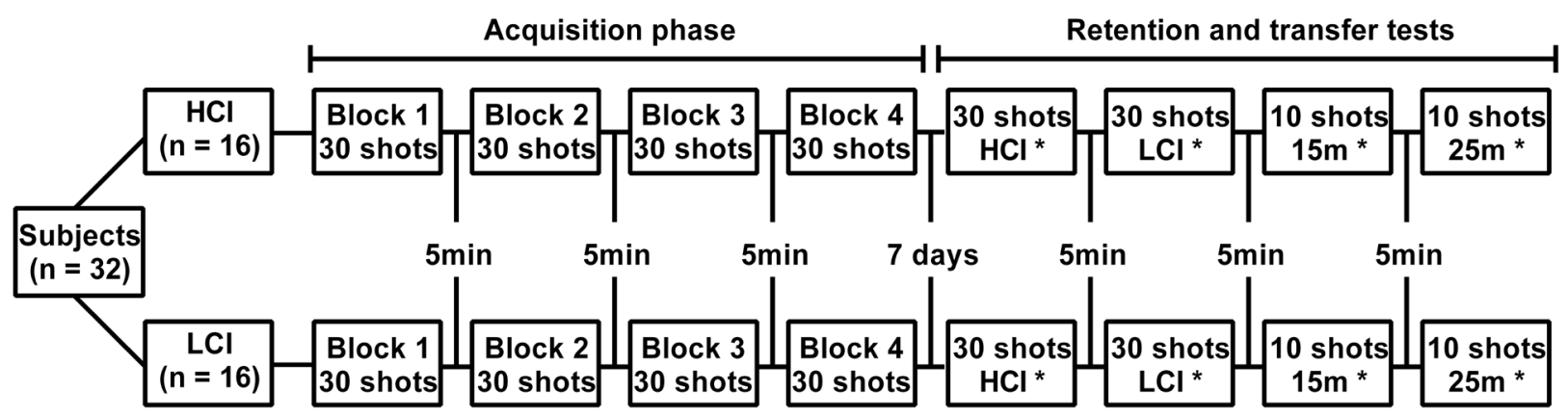

$\mathrm{HCl}$ - high contextual interference group, $\mathrm{LCl}$ - low contextual interference group; * test orders randomized between subjects

Figure 2. Experimental design

transfer tests were performed. Prior to each test, three familiarization shots were allowed in each position. The test itself consisted of thirty shots, ten in each position. Two retention tests were performed during which the HCI group changed positions before each shot, while the LCI group shot 10 times in the same position before changing to the next one. Position and test orders were also randomized between subjects.

The transfer test was performed with different target distances. Ten shots were attempted at 15 and 25 meters from the target. Both transfer tests were performed in the ST position. Figure 2 presents the study timeline.

\section{Rifle and target}

The rifle used in this research was a Phanton 1000 (Crosman) with a length of $1.13 \mathrm{~m}$, a weight of $2.25 \mathrm{~kg}$, a shot by shot reload system, and $4.5 \mathrm{~mm}$ pellets (Gamo Match, .177 caliber). A new shooting spring (45kg GR, FNA), estimated to provide four hundred thousand shots without performance loss, was set in the rifle before the study to guarantee quality aiming and linear displacement of the pellets throughout the study protocol. The rifle mean shooting velocity was $273 \mathrm{~m} / \mathrm{s}$, measured with a ballistic chronograph (Alpha Chrony).

The targets (figure 3) were printed on paper sheets, A4 size, containing score values ranging from 1 (outer circle) to 10 (inner circle). Each point score was awarded based on the following distances from the center of the target: 1 point $-14.5 \mathrm{~cm} ; 2$ points $-13.0 \mathrm{~cm}$; 3 points $-11.5 \mathrm{~cm}$; 4 points $-10 \mathrm{~cm}$; 5 points $-8.5 \mathrm{~cm}$; 6 points $7.0 \mathrm{~cm} ; 7$ points $-5.5 \mathrm{~cm} ; 8$ points $-4.0 \mathrm{~cm} ; 9$ points $2.5 \mathrm{~cm} ; 10$ points $-1 \mathrm{~cm}$. The target was replaced after five shots, and the scores representing the sum of each thirty-shot block were computed for analysis. When the pellet hit exactly the line between scores, the higher value was considered.

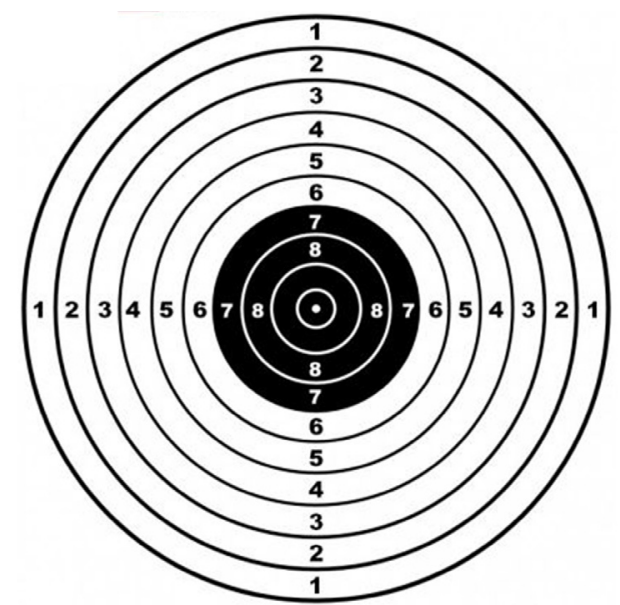

Figure 3. Target representation

A wooden board was used behind the target to prevent pellets from rebounding. The target was positioned $140 \mathrm{~cm}$ from the ground in the ST position, $70 \mathrm{~cm}$ in the FT position, and $30 \mathrm{~cm}$ in the LD position. The ST position target height followed the Olympic and International Shooting Sport Federation standard.

\section{Shooting procedures}

Before the acquisition phase, participants received verbal instructions and a visual demonstration of the appropriate technique and postures. One familiarization shot was allowed in each position. Participants were instructed to aim at the target and take the shot, not taking longer than seven seconds after receiving the rifle in their hands. An experienced researcher reloaded the gun and handed it back to the participant after each shot. This reloading procedure took approximately 4-8 seconds, hence the interval between shots being around 11-15 seconds. A two-minute break was allowed after every 5 shots. Participants rested for 5 minutes after completing the thirty-shot block.

The shots were performed indoors to control environmental changes and provide similar conditions for 
every participant. A duct tape line was used to determine the target distance in the different phases $(10 \mathrm{~m}$ in acquisition and retention, $15 \mathrm{~m}$ and $25 \mathrm{~m}$ in transfer). There were no gun sights to help shooting in any condition or a tripod support.

\section{Statistical analysis}

Data, with normality verified by the Shapiro-Wilk test, are described as means and standard deviations. Comparisons between groups and phases were carried out by two way ANOVA, and the Tukey post-hoc test was used when necessary. The Student's t Test for unrelated measures was performed to compare groups in the transfer tests. All data were processed in SPSS (v. 20.0), and significance was set at 5\%.

\section{Ethical approval}

The research related to human use has been complied with all the relevant national regulations and institutional policies, has followed the tenets of the Declaration of Helsinki, and has been approved by the authors' institutional review board or an equivalent committee.

The research was approved by the local University ethics committee (Res. 466/12, n ${ }^{\circ} 20386013.9 .0000$. 5231).

\section{Informed consent}

All participants were informed about all procedures and signed an informed consent form prior to participation.

\section{Results}

A phase effect was verified $\left(F_{1,5}=3,22 ; p=0.009\right)$, showing performance enhancement after practice for both groups. Average mean scores for both groups in each block (B1, B2, B3, and B4) were $M=5.53$ ( $S D=$ $0.28), M=6.08(S D=0.22), M=6.14(S D=0.20)$, and $M=6.32(S D=0.19)$, respectively. For the retention tests, the LCI score was $M=6.08(S D=0.25)$, and the HCI score was $M=6.33(S D=0.22)$. The groups did not differ from each other $\left(F_{1,5}=0.733 ; p=0.40\right)$ since the average score for the LCI was $M=5.93(S D=0.26)$, while the HCI scored $M=6.23(S D=0.24)$. Interaction between groups and phases was also not verified $\left(F_{1,5}=0.314 ; p=0.904\right)$. Figure 4 presents the average shooting scores in each block for the retention and transfer tests in both groups.

The unrelated measures t-test presented equal performance between groups in transfer tests from both $15 \mathrm{~m}$

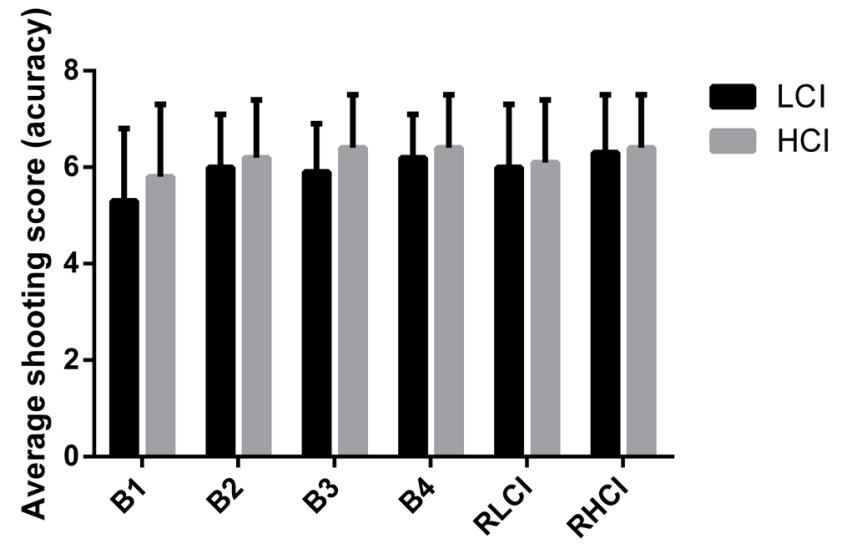

$\mathrm{LCl}$ - low contextual interference group, $\mathrm{HCl}$ - high contextual interference group, B1 - shooting block 1, B2 - shooting block 2, B3 - shooting block 3, B4 - shooting block 4, RLCl - low contextual interference retention test, $\mathrm{RHCl}$ - high contextual interference retention test

Figure 4. Mean and standard deviation of shooting scores for both groups in standing position in all experimental phases

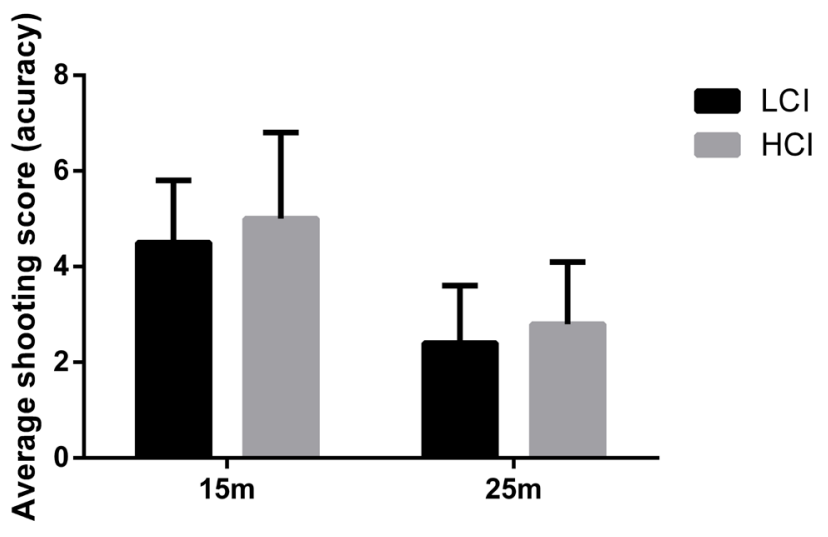

$\mathrm{LCl}$ - low contextual interference group, $\mathrm{HCl}$ - high contextual interference group, $15 \mathrm{~m}$ - transfer test 15 meters from target, $25 \mathrm{~m}$ - transfer test 25 meters from target

Figure 5. Mean and standard deviation of shooting scores for both groups in $15 \mathrm{~m}$ and $25 \mathrm{~m}$ transfer tests in standing position

$(t=-0.910 ; g l=26 ; p=0.372)$ and $25 \mathrm{~m}(t=-0.954$; $g l=26 ; p=0.349)$. The average shooting scores of transfer tests for both groups are shown in figure 5 .

\section{Discussion}

The main findings of this study were that practice enhanced shooting accuracy although a contextual interference effect was not established based on our data. There was insufficient evidence to support our initial hypotheses since accuracy was similar between groups during the acquisition phase, and the HCI group did not present significantly better scores in retention or transfer tests. Since developing the necessary musclearticular links and optimal isometric control to achieve 
success in a shooting task is a multifaceted process, these results should be viewed in the light of motor learning literature with consideration for motor control and neuroscience contributions in the subject.

The support for practice with greater CI as an aid to motor learning is originally based on the contrast that exists between the different movements performed during practice. These changes oblige participants to constantly mentally reconstruct an action plan during the acquisition phases, thus leading to better retention [1]. The planning of a motor skill promotes premature and extensive activation of the brain preand supplementary motor area which favors better longterm memory storage of that skill [10]. High contextual interference, therefore, seems to help in retention and movement planning since it provides greater stimulus for these processes during practice, which possibly reflects better performance and learning of the referred skill.

Brady's systematic review [8], on the other hand, pointed out that there is reduced applicability of the CI phenomenon in complex motor skills when compared to its effects on simpler, laboratory-based tasks. For example, $\mathrm{Wu}$ and colleagues [2] verified better retention and transfer in the HCI group when analyzing the single joint movement of extension and flexion at the elbow. Similarly, in producing a finger tapping sequence, Meira, Fairbrother and Perez [3] also found greater results in retention tests for the HCI group. The motor skills analyzed in the present study are more complex than the simple laboratory-based tasks described previously, which may explain why the CI effect was not well-established in our data. This assessment is also in alignment with Brady's investigation [8].

Other recent research, also comparing motor skills acquisition with CI manipulation [5, 6, 7, 11], did not verify positive results for random practice. Even though the task was very simple (two sequences of finger tapping) in the Neville and Trempe study [6], the varied practice group was not superior to the blocked group when analyzing retention tests. Sattelmeyer et al., in a systematic review [7], and Spruit et al., in their study [11], investigated surgical medical skill learning, while the study by Carter and Grahn [5] analyzed music playing with a clarinet. None verified better outcomes with random practice. These tasks, just like rifle shooting, demand high precision, attention, and fine movement adjustments, greatly differing from laboratory tasks that usually reinforce the positive effect of contextual interference manipulation. Therefore, these results are also in consonance with Brady's review [8].
The development of skilled shooting depends on a bilateral, synchronized, coordinated, motor action. One hand should stabilize the weapon to make it as motionless as possible, while the other should pull the trigger without any impact on final aim. This task imposes a challenge to the muscle-articular link synergies, since an eventual joint movement should be readily compensated by another in order to shoot precisely on target, requiring a well-established coordinative structure [9]. Therefore, developing this complex motor control cooperation that keeps all possible degrees of freedom under constraint may take longer practice periods depending on the subject's experience, previous motor repertory, and other internal characteristics. This complexity could explain the maintenance of data variability throughout the acquisition phase and that only six subjects of each group effectively enhanced accuracy. Even though the average scores presented a statistical difference favoring learning with practice, the mean changes were probably influenced by these 6 subjects. Since there was no effective learning of the entire group, the contextual interference effect might have been diminished, resulting in similar group scores, independent of the practice variability.

The similarity between groups despite varied CI may also have occurred due to the amount and duration of the acquisition phase, which was completed in one day and with only 120 shots. Since CI favors better long-term memory storage of a motor skill [10], a longer practice period could have made the difference more evident between the two forms of practice organization. Besides this limitation, it is possible that the different shooting positions were not challenging enough to characterize a high contextual interference protocol, as the isometric control of upper limbs is similar when shooting in standing, sitting, and lying. Nevertheless, our results are in accordance with Brady [8], also agreeing with other experimental research that did not find better learning of complex motor skills in practices with higher contextual interference [5, 11].

\section{Conclusions}

Both the low and high contextual interference groups demonstrated similar performance gains after practice, without variation between groups in the acquisition, retention, or transfer phases. Therefore, the initial hypotheses that the blocked practice (LCI) would lead to better results in the acquisition phase, while varied practice (HCI) would enable greater retention and transfer, were not verified in this study. 


\section{HUMAN MOVEMENT}

N.A. Moretto, A.J. Marcori, V.H.A. Okazaki, Contextual interference on rifle shooting

\section{Disclosure statement}

No author has any financial interest or received any financial benefit from this research.

\section{Conflict of interest}

The authors state no conflict of interest.

\section{References}

1. Shea JB, Morgan RL. Contextual interference effects on the acquisition, retention, and transfer of a motor skill. J Exp Psychol. 1979;5(2):179-187; doi: 10.1037/02787393.5.2.179.

2. Wu WFW, Young DE, Schandler SL, Meir G, Judy RLM, Perez J, et al. Contextual interference and augmented feedback: Is there an additive effect for motor learning? Hum Mov Sci. 2011;30(6):1092-1101; doi: 10.1016/j.humov.2011.02.004.

3. Meira CM, Fairbrother JT, Perez CR. Contextual interference and introversion/extraversion in motor learning. Percept Mot Skills. 2015;121(2):447-460; doi: 10.2466/23.PMS.121c20x6.

4. Pauwels L, Swinnen SP, Beets IAM. Contextual interference in complex bimanual skill learning leads to better skill persistence. PLoS One. 2014;9(6):e100906; doi: 10.1371/journal.pone.0100906.

5. Carter CE, Grahn JA. Optimizing music learning: Exploring how blocked and interleaved practice schedules affect advanced performance. Front Psychol. 2016;7: 1251; doi: 10.3389/fpsyg.2016.01251.

6. Neville K-M, Trempe M. Serial practice impairs motor skill consolidation. Exp Brain Res. 2017;235(9):26012613; doi: 10.1007/s00221-017-4992-6.

7. Sattelmayer M, Elsig S, Hilfiker R, Baer G. A systematic review and meta-analysis of selected motor learning principles in physiotherapy and medical education. BMC Med Educ. 2016;16(1):15; doi: 10.1186/s12909016-0538-z.

8. Brady F. The contextual interference effect and sport skills. Percept Mot Skills. 2008;106(2):461-472;doi: 10.2466/pms.106.2.461-472.

9. Tuller B, Turvey MT, Fitch HL. The Bernstein Perspective: II. The concept of muscle linkage or coordinative structures. In: Kelso JAS, editor. Human Motor Behavior, an Introduction. New Jersey: Lawrence Erlbaum Associates; 1982. p. 253-270.

10. Wright D, Verwey W, Buchanen J, Chen J, Rhee J, Immink M. Consolidating behavioral and neurophysiologic findings to explain the influence of contextual interference during motor sequence learning. Psychon Bull Rev. 2016:23(1),1-21, doi: 10.3758/s13423-0150887-3.

11. Spruit EN, Kleijweg L, Band GPH, Hamming JF. Varied practice in laparoscopy training: Beneficial learning stimulation or cognitive overload? Front Psychol. 2016:7; 685; doi: 10.3389/fpsyg.2016.00685. 